\title{
Almost Periodic Solution of a Discrete Commensalism System
}

\author{
Yalong Xue, ${ }^{1}$ Xiangdong Xie, ${ }^{1}$ Fengde Chen, ${ }^{1}$ and Rongyu Han ${ }^{2}$ \\ ${ }^{1}$ Department of Mathematics, Ningde Normal University, Ningde, Fujian 352100, China \\ ${ }^{2}$ College of Mathematics and Computer Science, Fuzhou University, Fuzhou, Fujian 350108, China
}

Correspondence should be addressed to Xiangdong Xie; ndsyxxd@163.com

Received 11 June 2015; Revised 27 September 2015; Accepted 1 October 2015

Academic Editor: Rigoberto Medina

Copyright (C) 2015 Yalong Xue et al. This is an open access article distributed under the Creative Commons Attribution License, which permits unrestricted use, distribution, and reproduction in any medium, provided the original work is properly cited.

\begin{abstract}
A nonautonomous discrete two-species Lotka-Volterra commensalism system with delays is considered in this paper. Based on the discrete comparison theorem, the permanence of the system is obtained. Then, by constructing a new discrete Lyapunov functional, a set of sufficient conditions which guarantee the system global attractivity are obtained. If the coefficients are almost periodic, there exists an almost periodic solution and the almost periodic solution is globally attractive.
\end{abstract}

\section{Introduction}

The study of the dynamic behaviors of discrete time systems governed by difference equation has become one of the most important topics in mathematic biology during the last decade. There are three main types of interaction between two species: (i) predator-prey, (ii) competition, and (iii) mutualism or symbiosis. Topics such as permanence and global attracitvity of these types were extensively investigated by scholars; see [1-9] and the references cited therein. However, commensalism, a typical relationship like epiphyte and plants with epiphyte, has few people to study it.

Sun and Wei [10] proposed the following commensalism system:

$$
\begin{aligned}
& \frac{d x}{d t}=r_{1} x\left(1-\frac{x}{K_{1}}+\alpha \frac{y}{K_{1}}\right), \\
& \frac{d y}{d t}=r_{2} y\left(1-\frac{y}{K_{2}}\right),
\end{aligned}
$$

where $r_{1}, r_{2}, K_{1}, K_{2}$, and $\alpha$ are all positive constants. By linearization of the system at positive equilibrium, they obtain that its corresponding linearization matrix has two negative eigenvalues; that is, the unique positive equilibrium is a stable node (Type I). Therefore, from First Degree Approximation Theory, we know that the unique positive equilibrium is asymptotically stable without any conditions.
Though much progress has been seen in the traditional Lotka-Volterra model, such models are not well studied in the sense that most results are continuous time cases related. Many authors have argued that the discrete time systems governed by difference equations are more approximate than the continuous ones when the populations have nonoverlapping generations. Discrete time models can also provide efficient computational models for numerical simulations. In a traditional continuous Lotka-Volterra cooperative system, if there is a positive equilibrium, the positive equilibrium is globally stable. But discrete Lotka-Volterra cooperative system cannot be permanent under any conditions. Therefore, the discrete systems are more difficult and complex to deal with compared to the continuous ones.

In this paper, we study the following discrete commensalism system:

$$
\begin{aligned}
& x(n+1)=x(n) \\
& \cdot \exp \left[r_{1}(n)\left(1-\frac{x\left(n-\tau_{1}\right)}{K_{1}(n)}+\alpha(n) \frac{y\left(n-\tau_{2}\right)}{K_{1}(n)}\right)\right], \\
& y(n+1)=y(n) \exp \left[r_{2}(n)\left(1-\frac{y\left(n-\tau_{3}\right)}{K_{2}(n)}\right)\right]
\end{aligned}
$$

where $x(n)$ and $y(n)$ represent the sizes or the densities of species $x$ and $y$ at $n$th generation, respectively. Parameters $r_{i}(n), K_{i}(n)(i=1,2)$, and $\alpha(n)$ are bounded nonnegative 
almost periodic sequences (for the definition, see Section 4) such that

$$
\begin{aligned}
& 0<r_{i}^{L} \leq r_{i}(n) \leq r_{i}^{M}, \\
& 0<K_{i}^{L} \leq K_{i}(n) \leq K_{i}^{M},
\end{aligned}
$$

$$
i=1,2 \text {, }
$$

$$
0<\alpha^{L} \leq \alpha(n) \leq \alpha^{M}, \quad n \in Z
$$

Here, denote the sets of integers and nonnegative integers by $Z$ and $Z^{+}$, respectively, and $\tau=\max \left\{\tau_{1}, \tau_{2}, \tau_{3}\right\}$. For any bounded sequence $g(n)$ defined on $Z$, denote $g^{M}=$ $\sup _{n \in Z} g(n)$ and $g^{L}=\inf _{n \in Z} g(n)$.

For biological reasons, we consider system (2) with the following initial conditions:

$$
(x(\theta), y(\theta))=(\varphi(\theta), \psi(\theta))>0, \quad \theta \in N[-\tau, 0] .
$$

It is not difficult to see that solutions of (2) are well defined for all $n \geq 0$ and satisfy $x(n)>0$.

The organization of this paper is as follows. In the next section, we show that (2) is permanent. In Section 3, a set of conditions which ensure that (2) is globally attractive are obtained. In Section 4, we will show that the almost periodic solutions are globally attractive. In Section 5, an example together with its numeric simulation is given to illustrate the feasibility of the main result.

\section{Permanence}

We first introduce some lemma.

Lemma 1 (see [11]). Assume that $\{x(n)\}$ satisfies $x(n)>0$ and for $n \in\left[N_{0},+\infty\right], x(n+1) \leq x(n) \exp \{r(n)(1-a x(n))\}$, where $a$ is a positive constant. Then

$$
\limsup _{n \rightarrow+\infty}(n) \leq \frac{1}{a r^{M}} \exp \left(r^{M}-1\right)
$$

Lemma 2 (see [11]). Assume that $\{x(n)\}$ satisfies $x(n+1) \geq$ $x(n) \exp \{r(n)(1-a x(n))\}, n \geq N_{0}$, limsup $_{n \rightarrow+\infty} x(n) \leq x^{*}$, and $x\left(N_{0}\right)>0$, where $a$ is a positive constant such that $a x^{*}>1$ and $N_{0} \in N$. Then

$$
\liminf _{n \rightarrow+\infty} x(n) \geq \frac{1}{a} \exp \left(r^{M}\left(1-a x^{*}\right)\right)
$$

Theorem A. For every positive solution $(x(n), y(n))$ of system (2), one has

$$
\begin{aligned}
& m_{1} \leq \liminf _{n \rightarrow+\infty} x(n) \leq \limsup _{n \rightarrow+\infty} x(n) \leq M_{1}, \\
& m_{2} \leq \liminf _{n \rightarrow+\infty} y(n) \leq \limsup _{n \rightarrow+\infty} y(n) \leq M_{2},
\end{aligned}
$$

where

$$
\begin{aligned}
M_{2} & =\frac{K_{2}^{M}}{r_{2}^{M}} \exp \left(r_{2}^{M}\left(\tau_{3}+1\right)-1\right), \\
m_{2} & =\frac{K_{2}^{L}}{\exp \left(-P \tau_{3}\right)} \exp \left[r_{2}^{M}\left(1-\frac{\exp \left(-P \tau_{3}\right)}{K_{2}^{L}} M_{2}\right)\right], \\
M_{1} & =\frac{K_{1}^{M}}{r_{1}^{M}} \exp \left(Q\left(\tau_{1}+1\right)-1\right), \\
m_{1} & =\frac{K_{1}^{L} T}{\exp \left(-R \tau_{1}\right)} \exp \left[r_{1}^{M}\left(T-\frac{\exp \left\{-R \tau_{1}\right\}}{K_{1}^{L}} M_{1}\right)\right], \\
P & =r_{2}^{M}\left(1-\frac{M_{2}}{K_{2}^{L}}\right), \\
Q & =r_{1}^{M}\left(1+\frac{\alpha^{M} M_{2}}{K_{1}^{L}}\right), \\
T & =1+\frac{\alpha^{L} m_{2}}{K_{1}^{M}}, \\
R & =r_{1}^{M}\left(T-\frac{M_{1}}{K_{1}^{L}}\right) .
\end{aligned}
$$

Proof of Theorem A. Let $(x(n), y(n))$ be an arbitrary solution of system (2). From the second equation of system (2), it follows that

$$
y(n+1) \leq y(n) \exp \left(r_{2}^{M}\right)
$$

From the above inequality, one has

$$
y\left(n-\tau_{3}\right) \geq y(n) \exp \left(-r_{2}^{M} \tau_{3}\right) .
$$

Substituting (10) into the second equation of (2), it follows that

$$
\begin{aligned}
& y(n+1) \\
& \quad \leq y(n) \exp \left[r_{2}(n)\left(1-\frac{\exp \left(-r_{2}^{M} \tau_{3}\right)}{K_{2}(n)} y(n)\right)\right] \\
& \quad \leq y(n) \exp \left[r_{2}(n)\left(1-\frac{\exp \left\{-r_{2}^{M} \tau_{3}\right\}}{K_{2}^{M}} y(n)\right)\right] .
\end{aligned}
$$

From Lemma 1 and (11), one has

$$
\limsup _{n \rightarrow+\infty} y(n) \leq \frac{K_{2}^{M}}{r_{2}^{M}} \exp \left(r_{2}^{M}\left(\tau_{3}+1\right)-1\right) \doteq M_{2} \text {. }
$$

For any positive constant $\varepsilon$ small enough, it follows from (12) that there exists a $N_{1}>0$ such that, for all $n \geq N_{1}$,

$$
y(n)<M_{2}+\varepsilon
$$


It follows from (13) and the second equation of (2), for $n \geq$ $N_{1}+\tau_{3}$, that

$$
\begin{aligned}
y(n+1) & \geq y(n) \exp \left[r_{2}(n)\left(1-\frac{M_{2}+\varepsilon}{K_{2}(n)}\right)\right] \\
& \geq y(n) \exp \left[r_{2}(n)\left(1-\frac{M_{2}+\varepsilon}{K_{2}^{L}}\right)\right] .
\end{aligned}
$$

Noting the fact that $(\exp (x-1)) / x \geq 1$, for $x>0$, we obtain

$$
\begin{aligned}
1-\frac{M_{2}+\varepsilon}{K_{2}^{L}} & <1-\frac{M_{2}}{K_{2}^{L}} \\
& =1-\frac{K_{2}^{M}}{K_{2}^{L}} \frac{\exp \left(r_{2}^{M}\left(\tau_{3}+1\right)-1\right)}{r_{2}^{M}} \leq-\tau_{3} \\
& <0 .
\end{aligned}
$$

Therefore, from (14) and (15), we have

$$
\begin{aligned}
y(n+1) & \geq y(n) \exp \left[r_{2}^{M}\left(1-\frac{M_{2}+\varepsilon}{K_{2}^{L}}\right)\right] \\
& \doteq y(n) \exp \left(P_{\varepsilon}\right) .
\end{aligned}
$$

By using (16), one could easily obtain that

$$
y\left(n-\tau_{3}\right) \leq y(n) \exp \left(-P_{\varepsilon} \tau_{3}\right) .
$$

Substituting (17) into the second equation of (2), we have $y(n+1)$

$$
\begin{aligned}
& \geq y(n) \exp \left[r_{2}(n)\left(1-\frac{\exp \left(-P_{\varepsilon} \tau_{3}\right)}{K_{2}(n)} y(n)\right)\right] \\
& \geq y(n) \exp \left[r_{2}(n)\left(1-\frac{\exp \left(-P_{\varepsilon} \tau_{3}\right)}{K_{2}^{L}} y(n)\right)\right] .
\end{aligned}
$$

Noting that $P_{\varepsilon}<0$,

$$
\begin{aligned}
& \frac{\exp \left(-P_{\varepsilon} \tau_{3}\right)}{K_{2}^{L}} M_{2} \\
& \quad=\exp \left(-P_{\varepsilon} \tau_{3}\right) \frac{K_{2}^{M}}{K_{2}^{L}} \frac{\exp \left(r_{2}^{M}\left(\tau_{3}+1\right)-1\right)}{r_{2}^{M}}>1+\tau_{3} \\
& >1 .
\end{aligned}
$$

Thus, according to Lemma 2 and (18), one has

$$
\begin{aligned}
& \liminf _{n \rightarrow+\infty} y(n) \\
& \quad \geq \frac{K_{2}^{L}}{\exp \left(-P_{\varepsilon} \tau_{3}\right)} \exp \left[r_{2}^{M}\left(1-\frac{\exp \left(-P_{\varepsilon} \tau_{3}\right)}{K_{2}^{L}} M_{2}\right)\right] .
\end{aligned}
$$

Setting $\varepsilon \rightarrow 0$, it follows that

$$
\begin{aligned}
& \liminf _{n \rightarrow+\infty} y(n) \\
& \quad \geq \frac{K_{2}^{L}}{\exp \left(-P \tau_{3}\right)} \exp \left[r_{2}^{M}\left(1-\frac{\exp \left(-P \tau_{3}\right)}{K_{2}^{L}} M_{2}\right)\right] \\
& \quad \doteq m_{2},
\end{aligned}
$$

where $P=r_{2}^{M}\left(1-M_{2} / K_{2}^{L}\right)$.
For the above $\varepsilon$, there exists a $N_{2}>N_{1}$ such that, for all $n>N_{2}$,

$$
m_{2}-\varepsilon<y(n)<M_{2}+\varepsilon
$$

From the first equation of system (2) and (22), for all $n-\tau_{2}>$ $N_{2}$,

$$
\begin{aligned}
x(n+1) & \leq x(n) \exp \left[r_{1}(n)\left(1+\alpha^{M} \frac{M_{2}+\varepsilon}{K_{1}(n)}\right)\right] \\
& \leq x(n) \exp \left[r_{1}^{M}\left(1+\alpha^{M} \frac{M_{2}+\varepsilon}{K_{1}^{L}}\right)\right] \\
& \doteq x(n) \exp \left(Q_{\varepsilon}\right) .
\end{aligned}
$$

From the above inequality, we have

$$
x\left(n-\tau_{1}\right) \geq x(n) \exp \left(-Q_{\varepsilon} \tau_{1}\right) .
$$

Substituting (24) into the first equation of (2), it follows that

$$
\begin{aligned}
& x(n+1) \leq x(n) \exp \left[r_{1}(n)\right. \\
& \left.\cdot\left(1+\alpha^{M} \frac{M_{2}+\varepsilon}{K_{1}^{L}}-\frac{\exp \left(-Q_{\varepsilon} \tau_{1}\right)}{K_{1}(n)} x(n)\right)\right] \leq x(n) \\
& \cdot \exp \left[r_{1}(n)\right. \\
& \left.\cdot\left(1+\alpha^{M} \frac{M_{2}+\varepsilon}{K_{1}^{L}}-\frac{\exp \left(-Q_{\varepsilon} \tau_{1}\right)}{K_{1}^{M}} x(n)\right)\right]=x(n) \\
& \cdot \exp \left[\frac{Q_{\varepsilon}}{r_{1}^{M}} r_{1}(n)\left(1-\frac{r_{1}^{M} \exp \left(-Q_{\varepsilon} \tau_{1}\right)}{Q_{\varepsilon} K_{1}^{M}} x(n)\right)\right] .
\end{aligned}
$$

From Lemma 1 and (25), we have

$$
\limsup _{n \rightarrow+\infty}(n) \leq \frac{K_{1}^{M}}{r_{1}^{M}} \exp \left(Q_{\varepsilon}\left(\tau_{1}+1\right)-1\right) .
$$

Setting $\varepsilon \rightarrow 0$, it follows that

$$
\limsup _{n \rightarrow+\infty}(n) \leq \frac{K_{1}^{M}}{r_{1}^{M}} \exp \left(Q\left(\tau_{1}+1\right)-1\right) \doteq M_{1},
$$

where $Q=r_{1}^{M}\left(1+\alpha^{M}\left(M_{2} / K_{1}^{L}\right)\right)$.

There exists a $N_{3}>N_{2}$ such that, for all $n \geq N_{3}$,

$$
x(n)<M_{1}+\varepsilon
$$

It follows from (28) and the first equation of (2), for $n>N_{3}+$ $\tau_{1}$, that

$$
\begin{aligned}
& x(n+1) \\
& \quad \geq x(n) \exp \left[r_{1}(n)\left(1+\alpha \frac{m_{2}-\varepsilon}{K_{1}^{M}}-\frac{M_{1}+\varepsilon}{K_{1}^{L}}\right)\right] .
\end{aligned}
$$


Noting the fact that $(\exp (x-1)) / x \geq 1$, for $x>0$, we obtain

$$
\begin{aligned}
1+\alpha^{L} \frac{m_{2}}{K_{1}^{M}}-\frac{M_{1}}{K_{1}^{L}}= & \left(1+\alpha^{L} \frac{m_{2}}{K_{1}^{M}}\right) \\
& -\frac{K_{1}^{M}}{K_{1}^{L}} \frac{\exp \left(Q\left(\tau_{1}+1\right)-1\right)}{r_{1}^{M}} \\
\leq & \left(1+\alpha^{L} \frac{m_{2}}{K_{1}^{M}}\right)-\frac{K_{1}^{M}}{K_{1}^{L}} \frac{Q\left(\tau_{1}+1\right)}{r_{1}^{M}} \\
\leq & \left(1+\alpha^{L} \frac{m_{2}}{K_{1}^{M}}\right) \\
& -\left(1+\alpha^{M} \frac{M_{2}}{K_{1}^{L}}\right)\left(\tau_{1}+1\right) \\
< & \left(1+\alpha^{L} \frac{m_{2}}{K_{1}^{M}}\right)-\left(1+\alpha^{M} \frac{M_{2}}{K_{1}^{L}}\right) \\
< & 0
\end{aligned}
$$

such that

$$
1+\alpha^{L} \frac{m_{2}-\varepsilon}{K_{1}^{M}}-\frac{M_{1}+\varepsilon}{K_{1}^{L}}<0, \quad \varepsilon>0 .
$$

Therefore, from the above inequality and (29), one has

$$
\begin{aligned}
& x(n+1) \\
& \quad \geq x(n) \exp \left[r_{1}^{M}\left(1+\alpha^{L} \frac{m_{2}-\varepsilon}{K_{1}^{M}}-\frac{M_{1}+\varepsilon}{K_{1}^{L}}\right)\right] \\
& \quad \doteq x(n) \exp \left(R_{\varepsilon}\right) .
\end{aligned}
$$

Using (32), we could easily obtain that

$$
x\left(n-\tau_{1}\right) \leq x(n) \exp \left(-R_{\varepsilon} \tau_{1}\right) .
$$

Substituting (33) into the first equation of (2), for $n \geq N_{3}+\tau_{1}$, deduces

$$
\begin{aligned}
& x(n+1) \geq x(n) \exp \left[r_{1}(n)\right. \\
& \left.\cdot\left(1+\alpha^{L} \frac{m_{2}-\varepsilon}{K_{1}^{M}}-\frac{\exp \left(-R_{\varepsilon} \tau_{1}\right)}{K_{1}^{L}} x(n)\right)\right]=x(n) \\
& \cdot \exp \left[\left(1+\alpha^{L} \frac{m_{2}-\varepsilon}{K_{1}^{M}}\right) r_{1}(n)\right. \\
& \left.\cdot\left(1-\frac{\exp \left(-R_{\varepsilon} \tau_{1}\right)}{K_{1}^{L}\left(1+\alpha^{L}\left(\left(m_{2}-\varepsilon\right) / K_{1}^{M}\right)\right)} x(n)\right)\right] .
\end{aligned}
$$

Noting the fact that $(\exp (x-1)) / x \geq 1$, for $x>0$, we obtain

$$
\begin{aligned}
& \frac{\exp \left(-R_{\varepsilon} \tau_{1}\right)}{K_{1}^{L}\left(1+\alpha^{L}\left(\left(m_{2}-\varepsilon\right) / K_{1}^{M}\right)\right)} M_{1} \\
& =\exp \left(-R_{\varepsilon} \tau_{1}\right) \frac{K_{1}^{M}}{K_{1}^{L}} \frac{\exp \left(Q\left(\tau_{1}+1\right)-1\right)}{r_{1}^{M}\left(1+\alpha^{L}\left(m_{2} / K_{1}^{M}\right)\right)} \\
& >\exp \left(-R_{\varepsilon} \tau_{1}\right) \frac{K_{1}^{M}}{K_{1}^{L}} \frac{Q\left(\tau_{1}+1\right)}{r_{1}^{M}\left(1+\alpha^{L}\left(m_{2} / K_{1}^{M}\right)\right)} \\
& =\exp \left(-R_{\varepsilon} \tau_{1}\right) \frac{K_{1}^{M}}{K_{1}^{L}} \frac{\left(1+\alpha^{M}\left(M_{2} / K_{1}^{L}\right)\right)}{\left(1+\alpha^{L}\left(m_{2} / K_{1}^{M}\right)\right)}\left(\tau_{1}+1\right) \\
& >1 .
\end{aligned}
$$

According to (34) and Lemma 2, one has

$$
\begin{aligned}
& \lim _{n \rightarrow+\infty} \inf (n) \geq \frac{K_{1}^{L}\left(1+\alpha^{L}\left(\left(m_{2}-\varepsilon\right) / K_{1}^{M}\right)\right)}{\exp \left(-R_{\varepsilon} \tau_{1}\right)} \\
& \quad \cdot \exp \left[r_{1}^{M}\left(1+\alpha^{L} \frac{m_{2}-\varepsilon}{K_{1}^{M}}-\frac{\exp \left\{-R_{\varepsilon} \tau_{1}\right\}}{K_{1}^{L}} M_{1}\right)\right] .
\end{aligned}
$$

Setting $\varepsilon \rightarrow 0$, it follows that

$$
\begin{aligned}
& \liminf _{n \rightarrow+\infty} x(n) \\
& \quad \geq \frac{K_{1}^{L} T}{\exp \left(-R \tau_{1}\right)} \exp \left[r_{1}^{M}\left(T-\frac{\exp \left\{-R \tau_{1}\right\}}{K_{1}^{L}} M_{1}\right)\right] \\
& \quad \doteq m_{1},
\end{aligned}
$$

where $T=1+\alpha^{L}\left(m_{2} / K_{1}^{M}\right)$ and $R=r_{1}^{M}\left(T-M_{1} / K_{1}^{L}\right)$. This completes the proof of Theorem A.

\section{Global Attractivity}

Assume that

$$
\begin{aligned}
& \frac{K_{2}^{M}}{K_{2}^{L}} \exp \left(r_{2}^{M}\left(1+\tau_{3}\right)-1\right)<\frac{2}{1+2 \tau_{3}}, \\
& \frac{2 r_{1}^{L} K_{1}^{L}}{r_{1}^{M} K_{1}^{M} M_{1}}>\frac{r_{1}^{M}}{2 K_{1}^{L}}\left(2 \tau_{1}+1\right)\left(2+\alpha^{M}\right)+\frac{\alpha^{M}}{m_{1}} .
\end{aligned}
$$

Theorem B. Assume that $\left(H_{1}\right)$ and $\left(H_{2}\right)$ hold; then system (2) is globally attractive; that is, for any positive solutions $(x(n), y(n))$ and $\left(x_{1}(n), y_{1}(n)\right)$ of system (2), one has

$$
\begin{aligned}
& \lim _{n \rightarrow+\infty}\left(x(n)-x_{1}(n)\right)=0, \\
& \lim _{n \rightarrow+\infty}\left(y(n)-y_{1}(n)\right)=0 .
\end{aligned}
$$

Proof of Theorem B. Let $(x(n), y(n))$ and $\left(x_{1}(n), y_{1}(n)\right)$ be any positive solutions of system (2).

According to the proof of Theorem 3.1 of [12], $\lim _{n \rightarrow+\infty}\left(y(n)-y_{1}(n)\right)=0$ holds under the condition 
$\left(H_{1}\right)$ and there exists a positive constant $M$ such that $\sum_{p=n_{0}+\tau}^{+\infty}\left(y(p)-y_{1}(p)\right)^{2} \leq M$. Now we just need to proof $\lim _{n \rightarrow+\infty}\left(x(n)-x_{1}(n)\right)=0$.

Condition $\left(H_{2}\right)$ implies that

$$
\begin{aligned}
& \frac{2 r_{1}^{L}}{K_{1}^{M} M_{1}} \\
& \quad-\frac{r_{1}^{M}}{K_{1}^{L}}\left(\frac{r_{1}^{M}}{K_{1}^{L}}\left(2 \tau_{1}+1\right)+\alpha^{M} \frac{r_{1}^{M}}{K_{1}^{L}}\left(\tau_{1}+\frac{1}{2}\right)+\frac{\alpha^{M}}{m_{1}}\right)
\end{aligned}
$$

$>0$.

Therefore, we can choose $\delta>0$ and $\varepsilon>0$ small enough such that

$$
\begin{aligned}
C_{\varepsilon} & \doteq \frac{2 r_{1}^{L}}{K_{1}^{M}\left(M_{1}+\varepsilon\right)}-\frac{r_{1}^{M}}{K_{1}^{L}}\left(\frac{r_{1}^{M}}{K_{1}^{L}}\left(2 \tau_{1}+1\right)\right. \\
& \left.+\alpha^{M} \frac{r_{1}^{M}}{K_{1}^{L}}\left(\tau_{1}+\frac{1}{2}\right)+\frac{\alpha^{M}}{\left(m_{1}-\varepsilon\right)}\right)>\delta .
\end{aligned}
$$

For any solutions $(x(n), y(n))$ and $\left(x_{1}(n), y_{1}(n)\right)$ of system $(2)$, it follows from Theorem $\mathrm{A}$ that

$$
\begin{aligned}
& m_{1} \leq \liminf _{n \rightarrow+\infty} x(n) \leq \limsup _{n \rightarrow+\infty} x(n) \leq M_{1}, \\
& m_{1} \leq \liminf _{n \rightarrow+\infty} x_{1}(n) \leq \lim _{n \rightarrow+\infty} \sup _{1}(n) \leq M_{1} .
\end{aligned}
$$

For the above $\varepsilon$, there exists an integer $n_{0}$ such that, for all $n \geq n_{0}$,

$$
\begin{aligned}
m_{1}-\varepsilon & \leq x(n), \\
x_{1}(n) & \leq M_{1}+\varepsilon .
\end{aligned}
$$

Using the mean value theorem, we get

$$
\ln x(n)-\ln x_{1}(n)=\frac{1}{\theta(n)}\left(x(n)-x_{1}(n)\right),
$$

where $\theta(n)$ lies between $x(n)$ and $x_{1}(n)$.

Let

$$
\begin{aligned}
W_{1}(n)= & \ln x(n)-\ln x_{1}(n) \\
& -\sum_{s=n-\tau_{1}}^{n-1} \frac{r_{1}\left(s+\tau_{1}\right)}{K_{1}\left(s+\tau_{1}\right)}\left(x(s)-x_{1}(s)\right) .
\end{aligned}
$$

Then, from system (2), we obtain

$$
\begin{aligned}
& W_{1}(n+1) \\
& =\ln x(n)-\ln x_{1}(n) \\
& \quad+\alpha(n) \frac{r_{1}(n)}{K_{1}(n)}\left(y\left(n-\tau_{2}\right)-y_{1}\left(n-\tau_{2}\right)\right) \\
& \quad-\sum_{s=n-\tau_{1}}^{n} \frac{r_{1}\left(s+\tau_{1}\right)}{K_{1}\left(s+\tau_{1}\right)}\left(x(s)-x_{1}(s)\right) .
\end{aligned}
$$

Therefore, we have

$$
\begin{aligned}
\Delta W_{1}(n) & =W_{1}(n+1)-W_{1}(n) \\
& =-\frac{r_{1}\left(n+\tau_{1}\right)}{K_{1}\left(n+\tau_{1}\right)}\left(x(n)-x_{1}(n)\right)+A(n),
\end{aligned}
$$

where $A(n)=\alpha(n)\left(r_{1}(n) / K_{1}(n)\right)\left(y\left(n-\tau_{2}\right)-y_{1}\left(n-\tau_{2}\right)\right)$.

Define

$$
V_{1}(n)=W_{1}^{2}(n)
$$

Then

$$
\begin{aligned}
& \Delta V_{1}(n)=W_{1}^{2}(n+1)-W_{1}^{2}(n)=\Delta W_{1}(n) \\
& \cdot\left(W_{1}(n+1)+W_{1}(n)\right)=-\frac{r_{1}\left(n+\tau_{1}\right)}{K_{1}\left(n+\tau_{1}\right)}(x(n) \\
& \left.-x_{1}(n)\right)\left(W_{1}(n+1)+W_{1}(n)\right)+A(n)\left(W_{1}(n+1)\right. \\
& \left.+W_{1}(n)\right)=-\frac{r_{1}\left(n+\tau_{1}\right)}{K_{1}\left(n+\tau_{1}\right)}\left(x(n)-x_{1}(n)\right) \\
& \left(2\left(\ln x(n)-\ln x_{1}(n)\right)-2\right. \\
& \cdot \frac{r_{1}(n)}{K_{1}(n)}\left(x\left(n-\tau_{1}\right)-x_{1}\left(n-\tau_{1}\right)\right) \\
& -2 \sum_{s=n+1-\tau_{1}}^{n-1} \frac{r_{1}\left(s+\tau_{1}\right)}{K_{1}\left(s+\tau_{1}\right)}\left(x(s)-x_{1}(s)\right) \\
& \left.-\frac{r_{1}\left(n+\tau_{1}\right)}{K_{1}\left(n+\tau_{1}\right)}\left(x(n)-x_{1}(n)\right)+A(n)\right)+A(n) \\
& \cdot\left(W_{1}(n+1)+W_{1}(n)\right)=-2 \frac{r_{1}\left(n+\tau_{1}\right)}{K_{1}\left(n+\tau_{1}\right)}(\ln x(n) \\
& \left.-\ln x_{1}(n)\right)\left(x(n)-x_{1}(n)\right)+2 \\
& \cdot \frac{r_{1}\left(n+\tau_{1}\right) r_{1}(n)}{K_{1}\left(n+\tau_{1}\right) K_{1}(n)}\left(x\left(n-\tau_{1}\right)-x_{1}\left(n-\tau_{1}\right)\right) \\
& \cdot\left(x(n)-x_{1}(n)\right)+2 \frac{r_{1}\left(n+\tau_{1}\right)}{K_{1}\left(n+\tau_{1}\right)} \\
& \cdot \sum_{s=n+1-\tau_{1}}^{n-1} \frac{r_{1}\left(s+\tau_{1}\right)}{K_{1}\left(s+\tau_{1}\right)}\left(x(s)-x_{1}(s)\right)\left(x(n)-x_{1}(n)\right) \\
& +\frac{r_{1}^{2}\left(n+\tau_{1}\right)}{K_{1}^{2}\left(n+\tau_{1}\right)}\left(x(n)-x_{1}(n)\right)^{2}+A(n) B(n),
\end{aligned}
$$

where $B(n)=W_{1}(n+1)+W_{1}(n)-\left(r_{1}\left(n+\tau_{1}\right) / K_{1}\left(n+\tau_{1}\right)\right)(x(n)-$ $\left.x_{1}(n)\right)$. 
Hence, from (43) and (48) and $2 a b \leq a^{2}+b^{2}$, we have $\Delta V_{1}(n)$

$$
\begin{aligned}
\leq & -2 \frac{r_{1}\left(n+\tau_{1}\right)}{K_{1}\left(n+\tau_{1}\right)} \frac{1}{\theta(n)}\left(x(n)-x_{1}(n)\right)^{2} \\
& +\frac{r_{1}^{2}\left(n+\tau_{1}\right)}{K_{1}^{2}\left(n+\tau_{1}\right)}\left(x(n)-x_{1}(n)\right)^{2} \\
& +\frac{r_{1}\left(n+\tau_{1}\right) r_{1}(n)}{K_{1}\left(n+\tau_{1}\right) K_{1}(n)}\left(x\left(n-\tau_{1}\right)-x_{1}\left(n-\tau_{1}\right)\right)^{2} \\
& +\frac{r_{1}\left(n+\tau_{1}\right) r_{1}(n)}{K_{1}\left(n+\tau_{1}\right) K_{1}(n)}\left(x(n)-x_{1}(n)\right)^{2} \\
& +\frac{r_{1}\left(n+\tau_{1}\right)}{K_{1}\left(n+\tau_{1}\right)} \sum_{s=n+1-\tau_{1}}^{n-1} \frac{r_{1}\left(s+\tau_{1}\right)}{K_{1}\left(s+\tau_{1}\right)}\left(x(s)-x_{1}(s)\right)^{2} \\
& +\frac{r_{1}\left(n+\tau_{1}\right)}{K_{1}\left(n+\tau_{1}\right)} \sum_{s=n+1-\tau_{1}}^{n-1} \frac{r_{1}\left(s+\tau_{1}\right)}{K_{1}\left(s+\tau_{1}\right)}\left(x(n)-x_{1}(n)\right)^{2} \\
+ & A(n) B(n) .
\end{aligned}
$$

Let

$$
\begin{aligned}
& V_{2}(n) \\
& =\sum_{s=n-\tau_{1}}^{n-1} \frac{r_{1}\left(s+2 \tau_{1}\right) r_{1}\left(s+\tau_{1}\right)}{K_{1}\left(s+2 \tau_{1}\right) K_{1}\left(s+\tau_{1}\right)}\left(x(s)-x_{1}(s)\right)^{2}, \\
& V_{3}(n)=\sum_{u=n}^{n-2+\tau_{1}} \frac{r_{1}\left(u+\tau_{1}\right)}{K_{1}\left(u+\tau_{1}\right)} \\
& \cdot \sum_{s=u+1-\tau_{1}}^{n-1} \frac{r_{1}\left(s+\tau_{1}\right)}{K_{1}\left(s+\tau_{1}\right)}\left(x(s)-x_{1}(s)\right)^{2} .
\end{aligned}
$$

Then

$$
\begin{aligned}
\Delta V_{2}(n) & \\
= & \frac{r_{1}\left(n+2 \tau_{1}\right) r_{1}\left(n+\tau_{1}\right)}{K_{1}\left(n+2 \tau_{1}\right) K_{1}\left(n+\tau_{1}\right)}\left(x(n)-x_{1}(n)\right)^{2} \\
& -\frac{r_{1}\left(n+\tau_{1}\right) r_{1}(n)}{K_{1}\left(n+\tau_{1}\right) K_{1}(n)}\left(x\left(n-\tau_{1}\right)-x_{1}\left(n-\tau_{1}\right)\right)^{2},
\end{aligned}
$$

$\Delta V_{3}(n)$

$$
\begin{aligned}
= & \frac{r_{1}\left(n+\tau_{1}\right)}{K_{1}\left(n+\tau_{1}\right)} \sum_{s=n+1}^{n-1+\tau_{1}} \frac{r_{1}\left(s+\tau_{1}\right)}{K_{1}\left(s+\tau_{1}\right)}\left(x(n)-x_{1}(n)\right)^{2} \\
& -\frac{r_{1}\left(n+\tau_{1}\right)}{K_{1}\left(n+\tau_{1}\right)} \sum_{s=n+1-\tau_{1}}^{n-1} \frac{r_{1}\left(s+\tau_{1}\right)}{K_{1}\left(s+\tau_{1}\right)}\left(x(s)-x_{1}(s)\right)^{2} .
\end{aligned}
$$

Define

$$
V_{4}(n)=V_{1}(n)+V_{2}(n)+V_{3}(n) .
$$

Then it follows from (49) and (51) that

$$
\begin{aligned}
& \Delta V_{4}(n) \leq-\frac{r_{1}\left(n+\tau_{1}\right)}{K_{1}\left(n+\tau_{1}\right)}\left(\frac{2}{\theta(n)}-\sum_{s=n-\tau_{1}}^{n+\tau_{1}} \frac{r_{1}\left(s+\tau_{1}\right)}{K_{1}\left(s+\tau_{1}\right)}\right) \\
& \cdot\left(x(n)-x_{1}(n)\right)^{2}+A(n) B(n),
\end{aligned}
$$

where

$$
\begin{aligned}
A(n) B(n)=\frac{2 \alpha(n) r_{1}(n)}{\theta(n) K_{1}(n)}\left(x(n)-x_{1}(n)\right)\left(y\left(n-\tau_{2}\right)\right. \\
\left.-y_{1}\left(n-\tau_{2}\right)\right)-\frac{2 \alpha(n) r_{1}^{2}(n)}{K_{1}^{2}(n)}\left(x\left(n-\tau_{1}\right)\right. \\
\left.-x_{1}\left(n-\tau_{1}\right)\right)\left(y\left(n-\tau_{2}\right)-y_{1}\left(n-\tau_{2}\right)\right) \\
-\frac{2 \alpha(n) r_{1}(n)}{K_{1}(n)} \sum_{s=n+1-\tau_{1}}^{n-1} \frac{r_{1}\left(s+\tau_{1}\right)}{K_{1}\left(s+\tau_{1}\right)}\left(x(s)-x_{1}(s)\right) \\
\cdot\left(y\left(n-\tau_{2}\right)-y_{1}\left(n-\tau_{2}\right)\right) \\
-\frac{\alpha(n) r_{1}\left(n+\tau_{1}\right) r_{1}(n)}{K_{1}\left(n+\tau_{1}\right) K_{1}(n)}\left(x(n)-x_{1}(n)\right) \\
\cdot\left(y\left(n-\tau_{2}\right)-y_{1}\left(n-\tau_{2}\right)\right) \\
+\frac{\alpha^{2}(n) r_{1}^{2}(n)}{K_{1}^{2}(n)}\left(y\left(n-\tau_{2}\right)-y_{1}\left(n-\tau_{2}\right)\right)^{2} .
\end{aligned}
$$

From $2 a b \leq a^{2}+b^{2}$ and (43), we have

$$
\begin{aligned}
& A(n) B(n) \leq \frac{\alpha(n) r_{1}(n)}{\theta(n) K_{1}(n)}\left(x(n)-x_{1}(n)\right)^{2} \\
& +\frac{\alpha(n) r_{1}(n)}{\theta(n) K_{1}(n)}\left(y\left(n-\tau_{2}\right)-y_{1}\left(n-\tau_{2}\right)\right)^{2} \\
& +\frac{\alpha(n) r_{1}^{2}(n)}{K_{1}^{2}(n)}\left(x\left(n-\tau_{1}\right)-x_{1}\left(n-\tau_{1}\right)\right)^{2} \\
& +\frac{\alpha(n) r_{1}^{2}(n)}{K_{1}^{2}(n)}\left(y\left(n-\tau_{2}\right)-y_{1}\left(n-\tau_{2}\right)\right)^{2} \\
& +\frac{\alpha(n) r_{1}(n)}{K_{1}(n)} \sum_{s=n+1-\tau_{1}}^{n-1} \frac{r_{1}\left(s+\tau_{1}\right)}{K_{1}\left(s+\tau_{1}\right)}\left(x(s)-x_{1}(s)\right)^{2} \\
& +\frac{\alpha^{2}(n) r_{1}^{2}(n)}{K_{1}^{2}(n)}\left(y\left(n-\tau_{2}\right)-y_{1}\left(n-\tau_{2}\right)\right)^{2} \\
& +\frac{\alpha(n) r_{1}(n)}{K_{1}(n)} \\
& \cdot \sum_{s=n+1-\tau_{1}}^{n-1} \frac{r_{1}\left(s+\tau_{1}\right)}{K_{1}\left(s+\tau_{1}\right)}\left(y\left(n-\tau_{2}\right)-y_{1}\left(n-\tau_{2}\right)\right)^{2} \\
& +\frac{\alpha(n) r_{1}\left(n+\tau_{1}\right) r_{1}(n)}{2 K_{1}\left(n+\tau_{1}\right) K_{1}(n)}\left(x(n)-x_{1}(n)\right)^{2}
\end{aligned}
$$




$$
\begin{aligned}
& +\frac{\alpha(n) r_{1}\left(n+\tau_{1}\right) r_{1}(n)}{2 K_{1}\left(n+\tau_{1}\right) K_{1}(n)}\left(y\left(n-\tau_{2}\right)\right. \\
& \left.-y_{1}\left(n-\tau_{2}\right)\right)^{2} .
\end{aligned}
$$

Let

$$
\begin{aligned}
& V_{5}(n)=\sum_{s=n-\tau_{1}}^{n-1} \frac{\alpha\left(s+\tau_{1}\right) r_{1}^{2}\left(s+\tau_{1}\right)}{K_{1}^{2}\left(s+\tau_{1}\right)}\left(x(s)-x_{1}(s)\right)^{2}, \\
& V_{6}(n)=\sum_{u=n}^{n-2+\tau_{1}} \frac{\alpha(u) r_{1}(u)}{K_{1}(u)} \\
& \cdot \sum_{s=u+1-\tau_{1}}^{n-1} \frac{r_{1}\left(s+\tau_{1}\right)}{K_{1}\left(s+\tau_{1}\right)}\left(x(s)-x_{1}(s)\right)^{2} .
\end{aligned}
$$

Then

$$
\begin{aligned}
& \Delta V_{5}(n) \\
& =\frac{\alpha\left(n+\tau_{1}\right) r_{1}^{2}\left(n+\tau_{1}\right)}{K_{1}^{2}\left(n+\tau_{1}\right)}\left(x(n)-x_{1}(n)\right)^{2} \\
& \quad-\frac{\alpha(n) r_{1}^{2}(n)}{K_{1}^{2}(n)}\left(x\left(n-\tau_{1}\right)-x_{1}\left(n-\tau_{1}\right)\right)^{2}, \\
& \Delta V_{6}(n) \\
& =\frac{r_{1}\left(n+\tau_{1}\right)}{K_{1}\left(n+\tau_{1}\right)} \sum_{s=n+1}^{n-1+\tau_{1}} \frac{\alpha(s) r_{1}(s)}{K_{1}(s)}\left(x(n)-x_{1}(n)\right)^{2} \\
& \quad-\frac{\alpha(n) r_{1}(n)}{K_{1}(n)} \sum_{s=u+1-\tau_{1}}^{n-1} \frac{r_{1}\left(s+\tau_{1}\right)}{K_{1}\left(s+\tau_{1}\right)}\left(x(s)-x_{1}(s)\right)^{2} .
\end{aligned}
$$

Define

$$
V(n)=V_{4}(n)+V_{5}(n)+V_{6}(n) .
$$

Then it follows from (53), (55), and (57) that

$$
\begin{aligned}
\Delta V(n) \leq & -C(n)\left(x(n)-x_{1}(n)\right)^{2} \\
& +D(n)\left(y\left(n-\tau_{2}\right)-y_{1}\left(n-\tau_{2}\right)\right)^{2},
\end{aligned}
$$

where

$$
\begin{aligned}
& C(n)=\left(\frac{2}{\theta(n)}-\sum_{s=n-\tau_{1}}^{n+\tau_{1}} \frac{r_{1}\left(s+\tau_{1}\right)}{K_{1}\left(s+\tau_{1}\right)}-\sum_{s=n+1}^{n+\tau_{1}} \frac{\alpha(s) r_{1}(s)}{K_{1}(s)}\right. \\
& \left.-\frac{\alpha(n) r_{1}(n)}{2 K_{1}(n)}\right) \frac{r_{1}\left(n+\tau_{1}\right)}{K_{1}\left(n+\tau_{1}\right)}-\frac{\alpha(n) r_{1}(n)}{\theta(n) K_{1}(n)} \\
& D(n)=\frac{\alpha(n) r_{1}(n)}{K_{1}(n)}\left(\frac{1}{\theta(n)}+\frac{r_{1}(n)}{K_{1}(n)}\right. \\
& \left.+\sum_{s=n+1-\tau_{1}}^{n} \frac{r_{1}\left(s+\tau_{1}\right)}{K_{1}\left(s+\tau_{1}\right)}+\frac{r_{1}\left(n+\tau_{1}\right)}{2 K_{1}\left(n+\tau_{1}\right)}\right) .
\end{aligned}
$$

Therefore, for all $n>n_{0}+\tau$,

$C(n) \geq C_{\varepsilon}$,

$$
D(n) \leq \frac{\alpha(n) r_{1}^{M}}{K_{1}^{L}}\left(\frac{1}{m_{1}-\varepsilon}+\frac{r_{1}^{M}}{K_{1}^{L}}\left(\tau_{1}+\frac{3}{2}\right)\right) \doteq D_{\varepsilon} .
$$

From (59) and (61), it follows that

$$
\begin{aligned}
\Delta V(n) \leq & -C_{\varepsilon}\left(x(n)-x_{1}(n)\right)^{2} \\
& +D_{\varepsilon}\left(y\left(n-\tau_{2}\right)-y_{1}\left(n-\tau_{2}\right)\right)^{2} \\
< & -\delta\left(x(n)-x_{1}(n)\right)^{2} \\
& +D_{\varepsilon}\left(y\left(n-\tau_{2}\right)-y_{1}\left(n-\tau_{2}\right)\right)^{2} .
\end{aligned}
$$

Summating both sides of the above inequalities from $n_{0}+\tau$ to $n$, we have

$$
\begin{aligned}
& \sum_{p=n_{0}+\tau}^{n}(V(p+1)-V(p)) \\
& \leq-\delta \sum_{p=n_{0}+\tau}^{n}\left(x(p)-x_{1}(p)\right)^{2} \\
& \quad+D_{\varepsilon} \sum_{p=n_{0}+\tau}^{n}\left(y\left(p-\tau_{2}\right)-y_{1}\left(p-\tau_{2}\right)\right)^{2},
\end{aligned}
$$

which implies

$$
\begin{aligned}
\sum_{p=n_{0}+\tau}^{n}\left(x(p)-x_{1}(p)\right)^{2} & \\
\leq & \frac{D_{\varepsilon}}{\delta} \sum_{p=n_{0}+\tau}^{n}\left(y\left(p-\tau_{2}\right)-y_{1}\left(p-\tau_{2}\right)\right)^{2} \\
+ & \frac{V\left(n_{0}+\tau\right)}{\delta} .
\end{aligned}
$$

It follows that $V_{i}\left(n_{0}+\tau\right), i=1,2,3,5,6$, are all bounded. Hence

$$
\begin{aligned}
& \sum_{p=n_{0}+\tau}^{n}\left(x(p)-x_{1}(p)\right)^{2} \\
& \leq \frac{D_{\varepsilon}}{\delta} \sum_{p=n_{0}+\tau}^{n}\left(y\left(p-\tau_{2}\right)-y_{1}\left(p-\tau_{2}\right)\right)^{2} \\
& \quad+\frac{V\left(n_{0}+\tau\right)}{\delta}<+\infty
\end{aligned}
$$

which means that

$$
\begin{aligned}
& \sum_{p=n_{0}+\tau}^{+\infty}\left(x(p)-x_{1}(p)\right)^{2} \\
& \leq \frac{D_{\varepsilon}}{\delta} \sum_{p=n_{0}+\tau}^{+\infty}\left(y\left(p-\tau_{2}\right)-y_{1}\left(p-\tau_{2}\right)\right)^{2} \\
& \quad+\frac{V\left(n_{0}+\tau\right)}{\delta}<+\infty .
\end{aligned}
$$


This implies that $\lim _{n \rightarrow+\infty}\left(x(n)-x_{1}(n)\right)^{2}=0$ or $\lim _{n \rightarrow+\infty}\left(x(n)-x_{1}(n)\right)=0$. This completes the proof of Theorem B.

\section{Almost Periodic Solution}

The main purpose of this paper is to study the existence of a globally attractive almost periodic sequence solution of system (2). First, we give some definitions and lemmas.

Definition 3 (see [13]). Sequence $x: Z \rightarrow R$ is called an almost periodic sequence if the $\varepsilon$-translation set of $x$,

$$
E\{\varepsilon, x\}=\{\tau \in Z:|x(n+\tau)-x(n)|<\varepsilon, \forall n \in Z\},
$$

is a relatively dense set in $Z$ for all $\varepsilon>0$; that is, for any given $\varepsilon>0$, there exists an integer $l(\varepsilon)>0$ such that each interval of length $l(\varepsilon)$ contains an integer $\tau \in E\{\varepsilon, x\}$ with

$$
|x(n+\tau)-x(n)|<\varepsilon, \quad \forall n \in Z .
$$

The integer $\tau$ is called an $\varepsilon$-translation number of $x(n)$.

Definition 4 (see [14]). Let $D$ be an open subset of $R^{m}$. A function $f: Z \times D \rightarrow R^{m}$ is said to be almost periodic in $n$ uniformly for $x \in D$, if, for any $\varepsilon>0$ and any compact set $S \subset D$, there exists a positive integer $l=l(\varepsilon, S)$, such that any interval of length $l$ contains an integer $\tau$, for which

$$
|f(n+\tau, x)-f(n, x)|<\varepsilon, \quad \forall(n, x) \in Z \times S .
$$

$\tau$ is called an $\varepsilon$-translation number of $f(n, x)$.

Definition 5 (see [15]). The hull of $f$, denoted by $H(f)$, is defined by

$$
\begin{gathered}
H(f)=\left\{g(n, x): \lim _{k \rightarrow \infty} f\left(n+\tau_{k}, x\right)\right. \\
=g(n, x) \text { uniformly on } Z \times S\}
\end{gathered}
$$

for some sequence $\left\{\tau_{k}\right\}$, where $S$ is any compact set in $D$.

Definition 6 (see [15]). Suppose that $X(n)=(x(n), y(n))$ is a solution of system (2). It is said to be a strictly positive solution in $Z$, if, for $n \in Z$,

$$
\begin{aligned}
& 0<\inf _{n \in Z} x(t) \leq \sup _{n \in Z} x(t)<\infty, \\
& 0<\inf _{n \in Z} y(t) \leq \sup _{n \in Z} y(t)<\infty .
\end{aligned}
$$

Lemma 7 (see [16]). Suppose that $\{x(n)\}$ is an almost periodic sequence if and only if, for any integer sequence $\left\{k_{i}^{\prime}\right\}$, there exists a subsequence $\left\{k_{i}\right\} \subset\left\{k_{i}^{\prime}\right\}$ such that the sequence $\left\{x\left(n+k_{i}\right)\right\}$ converges uniformly for all $n \in Z$ as $i \rightarrow \infty$. Furthermore, the limit sequence is also an almost periodic sequence.

From Theorem 3.4 in [17], we can easily obtain the following lemma.
Lemma 8. If each hull equation of system (2) has a unique strictly positive solution, then the almost periodic difference system (2) has a unique strictly positive almost periodic solution.

Under the consumption of $\left\{r_{i}(n)\right\},\left\{K_{i}(n)\right\}(i=1,2)$, and $\{\alpha(n)\}$ being bounded nonnegative almost periodic sequences, we have the following Theorem.

Theorem C. Assume that $\left(H_{1}\right)$ and $\left(H_{2}\right)$ hold; then the almost periodic difference system (2) admits a unique almost periodic sequence solution which is globally attractive.

Let $\left\{\xi_{k}\right\}$ be any integer value sequence such that $\xi_{k} \rightarrow \infty$ as $k \rightarrow \infty$. According to Lemma 7 , taking subsequence if necessary, we have $r_{i}\left(n+\xi_{k}^{i}\right) \rightarrow r_{i}^{*}(n), K_{i}\left(n+\xi_{k}^{i}\right) \rightarrow K_{i}^{*}(n)$, and $\alpha\left(n+\xi_{k}^{3}\right) \rightarrow \alpha^{*}(n)$ as $k \rightarrow \infty$ for $n \in Z$. Then the hull equation of (2) is as follows:

$$
\begin{aligned}
& x(n+1)=x(n) \exp \left[r_{1}^{*}(n)\right. \\
& \left.\cdot\left(1-\frac{x\left(n-\tau_{1}\right)}{K_{1}^{*}(n)}+\alpha^{*}(n) \frac{y\left(n-\tau_{2}\right)}{K_{1}^{*}(n)}\right)\right], \\
& y(n+1)=y(n) \exp \left[r_{2}^{*}(n)\left(1-\frac{y\left(n-\tau_{3}\right)}{K_{2}^{*}(n)}\right)\right] .
\end{aligned}
$$

By the almost periodic theory, we can conclude that if (2) satisfies $\left(H_{1}\right)$ and $\left(H_{2}\right)$, then the hull equation (72) also satisfies $\left(H_{1}\right)$ and $\left(H_{2}\right)$.

Proof of Theorem C. By Lemma 7, we only need to prove that each hull equation of (2) has a unique strictly positive solution. Suppose $(x(n), y(n))$ is any positive solution of hull equation of (72). First, we prove that the hull equation of (2) has at least one strictly positive solution. By Theorem A, we have

$$
\begin{aligned}
& m_{1} \leq \liminf _{n \rightarrow+\infty} x(n) \leq \limsup _{n \rightarrow+\infty} x(n) \leq M_{1}, \\
& m_{2} \leq \liminf _{n \rightarrow+\infty} y(n) \leq \limsup _{n \rightarrow+\infty} y(n) \leq M_{2},
\end{aligned}
$$

which gives

$$
\begin{aligned}
& 0<\inf _{n \in Z^{+}} x(n) \leq \sup _{n \in Z^{+}} x(n)<\infty, \\
& 0<\inf _{n \in Z^{+}} y(n) \leq \sup _{n \in Z^{+}} y(n)<\infty .
\end{aligned}
$$

Let $\varepsilon$ be an arbitrary small positive number. It follows from (73) that there exists a positive integer $N_{0}$ such that, for all $n>N_{0}$, we have $m_{1}-\varepsilon \leq x(n) \leq M_{1}+\varepsilon, m_{2}-\varepsilon \leq y(n) \leq$ $M_{2}+\varepsilon$. Define $x_{k}(n)=x\left(n+\tau_{k}\right), y_{k}(n)=y\left(n+\tau_{k}\right)$, for all $n>N_{0}+\tau-\tau_{k}, k \in Z^{+}$. For any positive integer $q$, it is easy to see that there exists a sequence $\left\{x_{k}(n): k \geq q\right\},\left\{y_{k}(n): k \geq\right.$ $q\}$ such that the sequence $\left\{x_{k}(n)\right\},\left\{y_{k}(n)\right\}$ has a subsequence, denoted by $\left\{x_{k}(n)\right\},\left\{y_{k}(n)\right\}$ again, converging on any finite 
interval of $Z$ as $k \rightarrow \infty$. Thus we have a sequence $\left\{x_{k}^{1}(n)\right\}$, $\left\{y_{k}^{1}(n)\right\}$ satisfying

$$
\begin{aligned}
& x_{k}(n) \longrightarrow x^{1}(n), \\
& y_{k}(n) \longrightarrow y^{1}(n),
\end{aligned}
$$

for $n \in Z$ as $k \longrightarrow \infty$.

Thus, combined with

$$
\begin{aligned}
& x_{k}(n+1)=x_{k}(n) \exp \left[r_{1}^{*}\left(n+\tau_{k}\right)\right. \\
& \left.\cdot\left(1-\frac{x_{k}\left(n-\tau_{1}\right)}{K_{1}^{*}\left(n+\tau_{k}\right)}+\alpha^{*}\left(n+\tau_{k}\right) \frac{y_{k}\left(n-\tau_{2}\right)}{K_{1}^{*}\left(n+\tau_{k}\right)}\right)\right], \\
& y_{k}(n+1)=y_{k}(n) \exp \left[r_{2}^{*}\left(n+\tau_{k}\right)\right. \\
& \left.\cdot\left(1-\frac{y_{k}\left(n-\tau_{3}\right)}{K_{2}^{*}\left(n+\tau_{k}\right)}\right)\right]
\end{aligned}
$$

gives us

$$
\begin{gathered}
x^{1}(n+1)=x^{1}(n) \exp \left[r_{1}^{*}(n)\right. \\
\left.\cdot\left(1-\frac{x^{1}\left(n-\tau_{1}\right)}{K_{1}^{*}(n)}+\alpha^{*}(n) \frac{y^{1}\left(n-\tau_{2}\right)}{K_{1}^{*}(n)}\right)\right], \\
y^{1}(n+1)=y^{1}(n) \exp \left[r_{2}^{*}(n)\left(1-\frac{y^{1}\left(n-\tau_{3}\right)}{K_{2}^{*}(n)}\right)\right] .
\end{gathered}
$$

We can easily see that $\left(\left(x^{1}(n)\right),\left(y^{1}(n)\right)\right)$ is a solution of (72) and $m_{1}-\varepsilon \leq x^{1}(n) \leq M_{1}+\varepsilon, m_{2}-\varepsilon \leq y^{1}(n) \leq M_{2}+\varepsilon$ for $n \in Z$. Since $\varepsilon$ is an arbitrary small positive number, it follows that $m_{1} \leq x^{1}(n) \leq M_{1}, m_{2} \leq y^{1}(n) \leq M_{2}$ for $n \in Z$; that is,

$$
\begin{aligned}
& 0<\inf _{n \in Z^{+}} x^{1}(n) \leq \sup _{n \in Z^{+}} x^{1}(n)<\infty, \\
& 0<\inf _{n \in Z^{+}} y^{1}(n) \leq \sup _{n \in Z^{+}} y^{1}(n)<\infty .
\end{aligned}
$$

Hence each hull equation of almost periodic difference system (2) has at least one strictly positive solution.

Now we prove the uniqueness of the strictly positive solution of each hull equation (72). Suppose that the hull equation has two arbitrary strictly positive solutions $\left(x^{*}(n), y^{*}(n)\right)$ and $\left(x_{1}^{*}(n), y_{1}^{*}(n)\right)$. From Theorem 1.3 in [12], we easily obtain that the hull equation of the second equation of (2) has a unique almost periodic solution which is globally attractive under condition $\left(H_{1}\right)$.

Similar to the proof of Theorem B, we define a Lyapunov functional

$$
V^{*}(n)=V_{1}^{*}(n)+V_{2}^{*}(n)+V_{3}^{*}(n)+V_{5}^{*}(n)+V_{6}^{*}(n),
$$

where

$$
\begin{aligned}
& V_{1}^{*}(n)=\left[\ln x^{*}(n)-\ln x_{1}^{*}(n)\right. \\
& \left.-\sum_{s=n-\tau_{1}}^{n-1} \frac{r_{1}^{*}\left(s+\tau_{1}\right)}{K_{1}^{*}\left(s+\tau_{1}\right)}\left(x^{*}(s)-x_{1}^{*}(s)\right)\right]^{2}, \\
& V_{2}^{*}(n)=\sum_{s=n-\tau_{1}}^{n-1} \frac{r_{1}^{*}\left(s+2 \tau_{1}\right) r_{1}^{*}\left(s+\tau_{1}\right)}{K_{1}^{*}\left(s+2 \tau_{1}\right) K_{1}^{*}\left(s+\tau_{1}\right)}\left(x^{*}(s)\right. \\
& \left.-x_{1}^{*}(s)\right)^{2} \text {, } \\
& V_{3}^{*}(n)=\sum_{u=n}^{n-2+\tau_{1}} \frac{r_{1}^{*}\left(u+\tau_{1}\right)}{K_{1}^{*}\left(u+\tau_{1}\right)} \\
& \cdot \sum_{s=u+1-\tau_{1}}^{n-1} \frac{r_{1}^{*}\left(s+\tau_{1}\right)}{K_{1}^{*}\left(s+\tau_{1}\right)}\left(x^{*}(s)-x_{1}^{*}(s)\right)^{2}, \\
& V_{5}^{*}(n)=\sum_{s=n-\tau_{1}}^{n-1} \frac{\alpha^{*}\left(s+\tau_{1}\right)\left(r_{1}^{*}\left(s+\tau_{1}\right)\right)^{2}}{\left(K_{1}^{*}\left(s+\tau_{1}\right)\right)^{2}}\left(x^{*}(s)\right. \\
& \left.-x_{1}^{*}(s)\right)^{2} \text {, } \\
& V_{6}^{*}(n)=\sum_{u=n}^{n-2+\tau_{1}} \frac{\alpha^{*}(u) r_{1}^{*}(u)}{K_{1}^{*}(u)} \\
& \cdot \sum_{s=u+1-\tau_{1}}^{n-1} \frac{r_{1}^{*}\left(s+\tau_{1}\right)}{K_{1}^{*}\left(s+\tau_{1}\right)}\left(x^{*}(s)-x_{1}^{*}(s)\right)^{2} .
\end{aligned}
$$

Calculating the difference of $V^{*}(n)$ along the solution of the hull equation (72) and the uniqueness of the second equation of (72), we have

$$
\Delta V^{*}(n) \leq-\delta\left(x^{*}(n)-x_{1}^{*}(n)\right)^{2}, \quad \text { for } n \in Z .
$$

From (81), we can see that $V^{*}(n)$ is a nonincreasing function on $Z$. Summating both sides of the above inequality from $n$ to 0 , we obtain

$$
\delta \sum_{p=n}^{0}\left(x^{*}(p)-x_{1}^{*}(p)\right)^{2} \leq V^{*}(n)-V^{*}(1)
$$

for $n<0$.

Note that $V^{*}(n)$ is bounded. Hence we have $\sum_{p=-\infty}^{0}\left(x^{*}(p)-\right.$ $\left.x_{1}^{*}(p)\right)^{2}<+\infty$, which implies that

$$
\lim _{n \rightarrow-\infty}\left(x^{*}(n)-x_{1}^{*}(n)\right)=0 .
$$

Denote $E=\left(1 / m_{1}+\tau_{1}\left(r_{1}^{M} / K_{1}^{L}\right)\right)^{2}+\left(\tau_{1}\left(\tau_{1}+1\right)(1+\right.$ $\left.\left.\alpha^{M}\right) / 2\right)\left(r_{1}^{M} / K_{1}^{L}\right)^{2}$. Let $\varepsilon$ be an arbitrary small positive number. It follows from (83) that there exists a positive integer $N_{1}>0$ such that $\left|x^{*}(n)-x_{1}^{*}(n)\right|<\sqrt{\varepsilon / E}, n<-N_{1}$. By the mean value theorem, we obtain $\ln x^{*}(n)-\ln x_{1}^{*}(n)=(1 / \theta(n))\left(x^{*}(n)-\right.$ $\left.x_{1}^{*}(n)\right)$, where $m_{1} \leq \theta(n) \leq M_{1}$. 
Hence, for $n<-N_{1}$, we have

$$
\begin{aligned}
& V_{1}^{*}(n) \leq\left(\frac{1}{\theta(n)}+\sum_{s=n-\tau_{1}}^{n-1} \frac{r_{1}^{*}\left(s+\tau_{1}\right)}{K_{1}^{*}\left(s+\tau_{1}\right)}\right)^{2} \\
& \cdot \max _{p \leq n}\left|x^{*}(p)-x_{1}^{*}(p)\right|^{2} \leq\left(\frac{1}{m_{1}}+\tau_{1} \frac{r_{1}^{M}}{K_{1}^{L}}\right)^{2} \frac{\varepsilon}{E}, \\
& V_{2}^{*}(n) \leq \tau_{1}\left(\frac{r_{1}^{M}}{K_{1}^{L}}\right)^{2} \max _{p \leq n}\left|x^{*}(p)-x_{1}^{*}(p)\right|^{2} \\
& \leq \tau_{1}\left(\frac{r_{1}^{M}}{K_{1}^{L}}\right)^{2} \frac{\varepsilon}{E} \\
& V_{3}^{*}(n) \leq \frac{\tau_{1}\left(\tau_{1}-1\right)}{2}\left(\frac{r_{1}^{M}}{K_{1}^{L}}\right)^{2} \max _{p \leq n}\left|x^{*}(p)-x_{1}^{*}(p)\right|^{2} \\
& \leq \frac{\tau_{1}\left(\tau_{1}-1\right)}{2}\left(\frac{r_{1}^{M}}{K_{1}^{L}}\right)^{2} \frac{\varepsilon}{E}, \\
& V_{5}^{*}(n) \leq \tau_{1} \alpha^{M}\left(\frac{r_{1}^{M}}{K_{1}^{L}}\right)^{2} \max _{p \leq n}\left|x^{*}(p)-x_{1}^{*}(p)\right|^{2} \\
& \leq \tau_{1} \alpha^{M}\left(\frac{r_{1}^{M}}{K_{1}^{L}}\right)^{2} \frac{\varepsilon}{E} \\
& V_{6}^{*}(n) \leq \frac{\tau_{1}\left(\tau_{1}-1\right)}{2} \alpha^{M}\left(\frac{r_{1}^{M}}{K_{1}^{L}}\right)^{2} \\
& \cdot \max _{p \leq n}\left|x^{*}(p)-x_{1}^{*}(p)\right|^{2} \leq \frac{\tau_{1}\left(\tau_{1}-1\right)}{2} \alpha^{M}\left(\frac{r_{1}^{M}}{K_{1}^{L}}\right)^{2} \\
& \cdot \frac{\varepsilon}{E} .
\end{aligned}
$$

It follows from (79) and the above inequalities that $V^{*}(n) \leq E(\varepsilon / E)=\varepsilon, n<-N_{1}$, so $\lim _{n \rightarrow-\infty} V^{*}(n)=0$. Note that $V^{*}(n)$ is a positive and nonincreasing function on $Z$. Then $V^{*}(n) \equiv 0$; that is, $x^{*}(n)=x_{1}^{*}(n)$, for all $n \in Z$. Therefore, each hull equation of system (2) has a unique strictly positive solution.

In view of the above discussion, any hull equation of system (2) has a unique strictly positive solution. By Lemma 8, the almost periodic difference system (2) has a unique strictly positive almost periodic solution $\left(\left(x^{*}(n)\right),\left(y^{*}(n)\right)\right)$. Similar to the proof of Theorem B, we can obtain $\lim _{n \rightarrow \infty}\left|x(n)-x^{*}(n)\right|=0$, where $x(n)$ is any positive solution of system (2). Therefore, the almost periodic system (2) has a unique strictly positive almost periodic solution which is globally attractive. This completes the proof of Theorem C.

\section{Numerical Simulations}

In this section, we give an example to check the feasibility of our result.

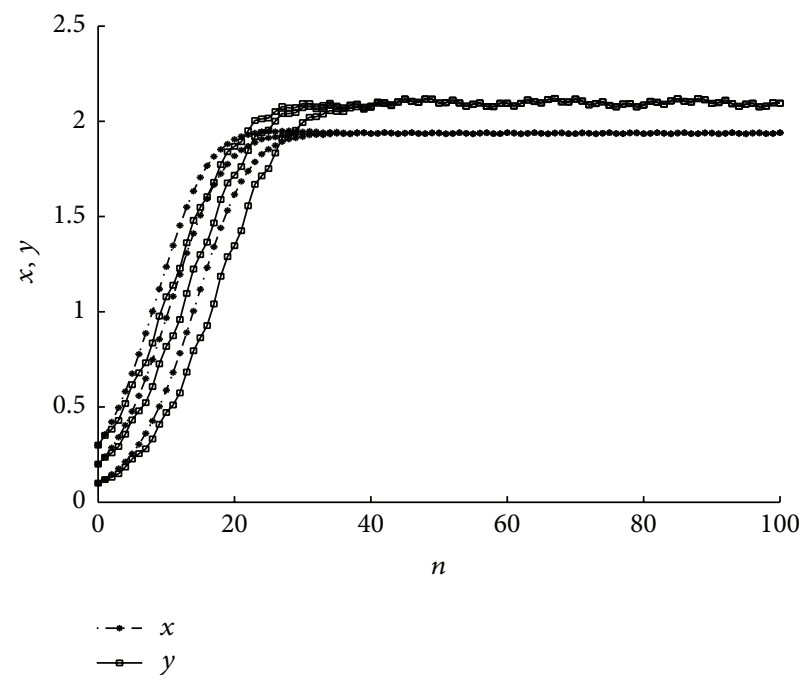

FIGURE 1: Dynamic behaviors of the solution $(x(n), y(n))$ of system (2) with initial conditions $(\varphi(\theta), \psi(\theta))=(0.1,0.3),(0.3,0.1)$, $(0.2,0.3)$ for $\theta=-2,-1,0$, respectively.

Example 9. Consider the following discrete commensalism system:

$$
\begin{aligned}
& x(n+1)=x(n) \exp \left[0 . 2 \left(1-\frac{x(n-1)}{2}\right.\right. \\
& \left.\left.+\frac{(0.03+0.01 \cos (\sqrt{3} n))}{2} y(n-2)\right)\right], \\
& y(n+1)=y(n) \exp [(0.17+0.07 \cos (\sqrt{2} n))(1 \\
& \left.\left.-\frac{y(n-1)}{2.1+0.1 \sin (\sqrt{3} n)}\right)\right] .
\end{aligned}
$$

It is easy to calculate that $M_{2} \approx 5.4498, m_{2} \approx 0.6249$ and $M_{1} \approx 5.2540, m_{1} \approx 0.8608$; then

$$
\begin{aligned}
& \frac{2}{1+2 \tau_{3}}-\frac{K_{2}^{M}}{K_{2}^{L}} \exp \left(r_{2}^{M}\left(1+\tau_{3}\right)-1\right) \approx 0.0127>0, \\
& \frac{2 r_{1}^{L} K_{1}^{L}}{r_{1}^{M} K_{1}^{M} M_{1}}-\frac{r_{1}^{M}}{2 K_{1}^{L}}\left(2 \tau_{1}+1\right)\left(2+\alpha^{M}\right)-\frac{\alpha^{M}}{m_{1}} \\
& \approx 0.0282>0 .
\end{aligned}
$$

It follows from Theorem $C$ that system (85) admits a unique almost periodic sequence solution which is globally attractive (see Figures 1 and 2).

\section{Discussion}

In this paper, we investigate an almost periodic discrete commensalism system with discrete delays. By constructing suitable Lyapunov functional, we study the global attractivity 

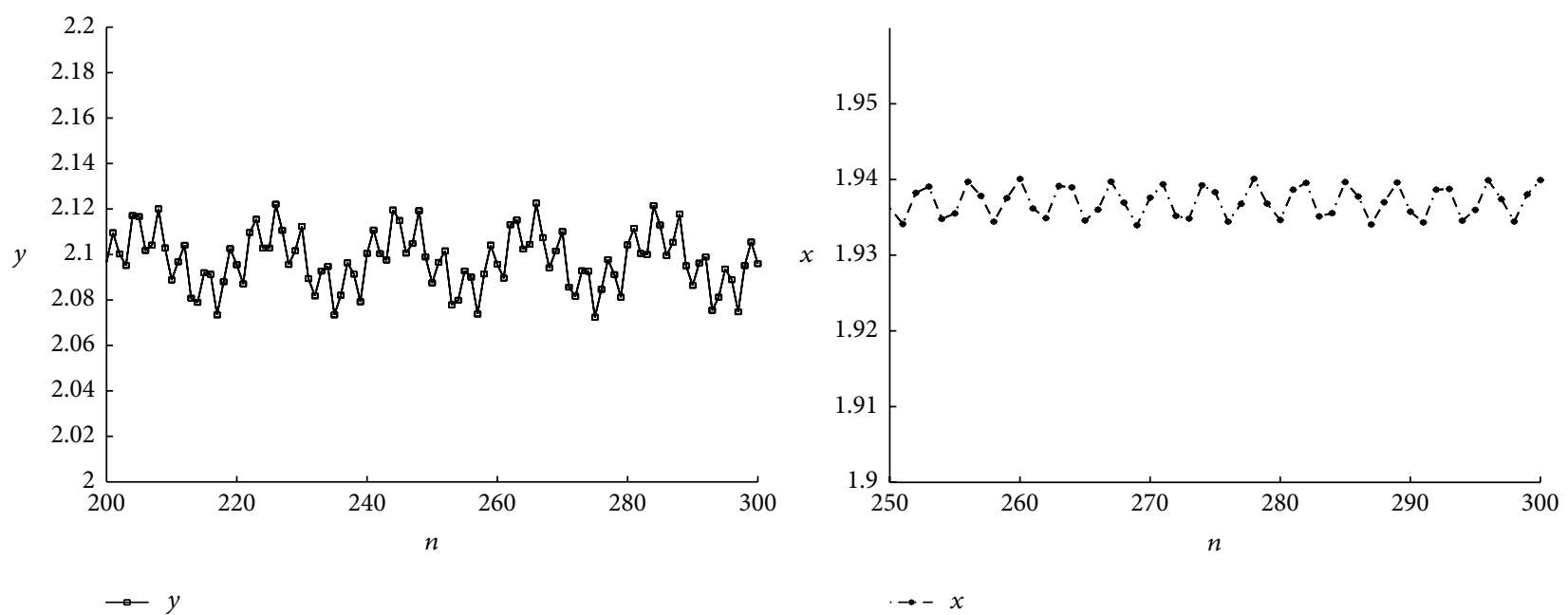

FIGURE 2: Almost periodic solution $(x(n), y(n))$ of system (2) with initial conditions $(\varphi(\theta), \psi(\theta))=(0.1,0.3),(0.3,0.1),(0.2,0.3)$ for $\theta=$ $-2,-1,0$, respectively.

of system (2). Using almost periodic functional hull theory, $\left(H_{1}\right)$ and $\left(H_{2}\right)$ are obtained for the existence of a unique globally attractive almost periodic sequence solution of system (2). Furthermore, $\tau_{1}$ and $\tau_{3}$ cannot be too large and at the same time $\tau_{2}$ has no influence on the global attractivity of the second species of system (2).

\section{Conflict of Interests}

The authors declare that there is no conflict of interests regarding the publication of this paper.

\section{Acknowledgment}

The research was supported by the Natural Science Foundation of Fujian Province (2013J01011 and 2013J01010).

\section{References}

[1] J. D. Murray, Mathematical Biology, Springer, New York, NY, USA, 2002.

[2] K. Yang, X. D. Xie, and F. D. Chen, "Global stability of a discrete mutualism model," Abstract and Applied Analysis, vol. 2014, Article ID 709124, 7 pages, 2014.

[3] F. D. Chen and X. D. Xie, Dynamic Behaviors of Cooperation Populations Models, Science Press, Beijing, China, 2014 (Chinese).

[4] L. S. Chen, X. Y. Song, and Z. Y. Lu, Mathematical Models and Methods in Ecology, Sichuan Science and Technology Press, Sichuan, China, 2003 (Chinese).

[5] X. D. Xie, F. D. Chen, K. Yang, and Y. L. Xue, "Global attractivity of an integrodifferential model of mutualism," Abstract and Applied Analysis, vol. 2014, Article ID 928726, 6 pages, 2014.

[6] L. Zhao, X. D. Xie, L. Y. Yang, and F. D. Chen, "Dynamic behaviors of a discrete Lotka-Volterra competition system with infinite delays and single feedback control," Abstract and Applied Analysis, vol. 2014, Article ID 867313, 19 pages, 2014.

[7] H. F. Huo and W. T. Li, "Permanence and global stability for nonautonomous discrete model of plankton allelopathy," Applied Mathematics Letters, vol. 17, no. 9, pp. 1007-1013, 2004.
[8] Z. Y. Lu and W. D. Wang, "Permanence and global attractivity for Lotka-Volterra difference systems," Journal of Mathematical Biology, vol. 39, no. 3, pp. 269-282, 1999.

[9] Z. F. Zhu, F. Xu, and Y. A. Li, "Mathematical analysis on commensalism Lotka-Volterra model of populations," Chongqing Institute of Technology (Natural Science), vol. 21, no. 10, pp. 5962, 2007.

[10] G. C. Sun and W. L. Wei, "The qualitative analysis of commensal symbiosis model of two populations," Mathematics: Theory \& Applications, vol. 23, no. 3, pp. 64-68, 2003.

[11] X. T. Yang, "Uniform persistence and periodic solutions for a discrete predator-prey system with delays," Journal of Mathematical Analysis and Applications, vol. 316, no. 1, pp. 161-177, 2006.

[12] Z. Li, M. A. Han, and F. D. Chen, "Almost periodic solutions of a discrete almost periodic logistic equation with delay," Applied Mathematics and Computation, vol. 232, pp. 743-751, 2014.

[13] A. M. Fink and G. Seifert, "Liapunov functions and almost periodic solutions for almost periodic systems," Journal of Differential Equations, vol. 5, no. 2, pp. 307-313, 1969.

[14] Y. Hamaya, "Existence of an almost periodic solution in a difference equation by Lyapunov functions," Nonlinear Studies, vol. 8, no. 3, pp. 373-379, 2001.

[15] S. N. Zhang, "Existence of almost periodic solution for defference systems," Annals of Differential Equations, vol. 16, no. 2, pp. 184-206, 2000.

[16] R. Yuan and J. L. Hong, "The existence of almost periodic solutions for a class of differential equations with piecewise constant argument," Nonlinear Analysis: Theory, Methods \& Applications, vol. 28, no. 8, pp. 1439-1450, 1997.

[17] S. N. Zhang and G. Zheng, "Almost periodic solutions of delay difference systems," Applied Mathematics and Computation, vol. 131, no. 2-3, pp. 497-516, 2002. 


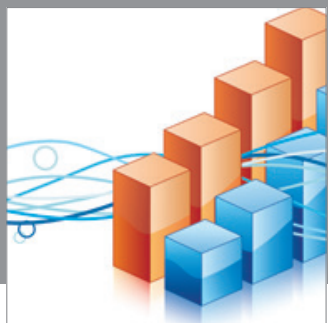

Advances in

Operations Research

mansans

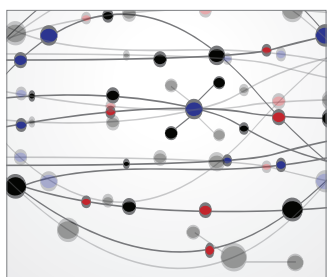

The Scientific World Journal
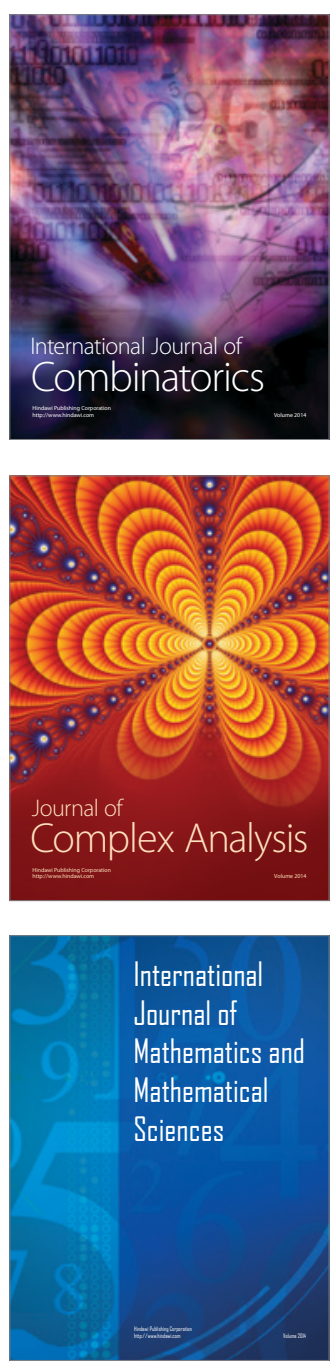
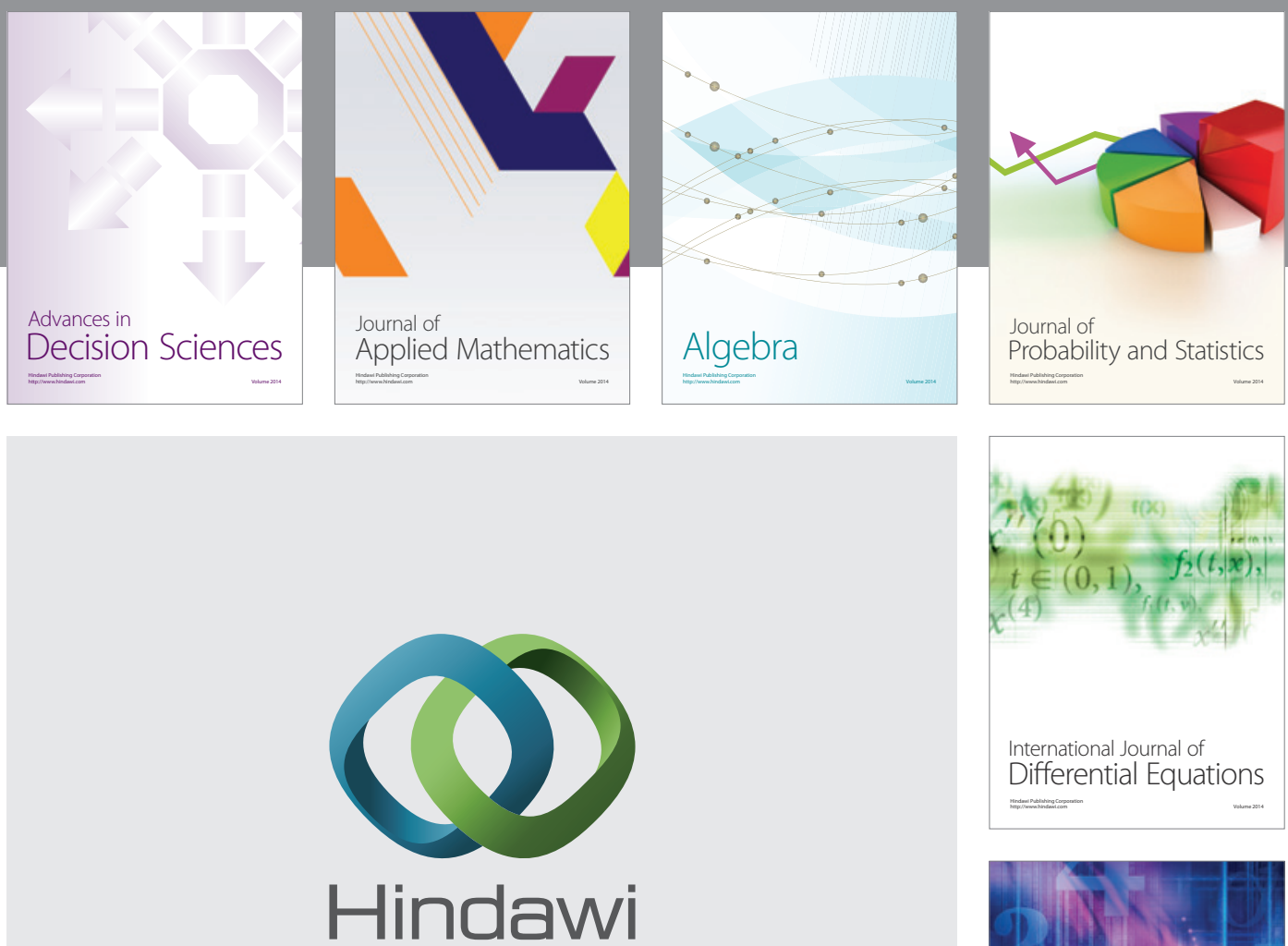

Submit your manuscripts at http://www.hindawi.com
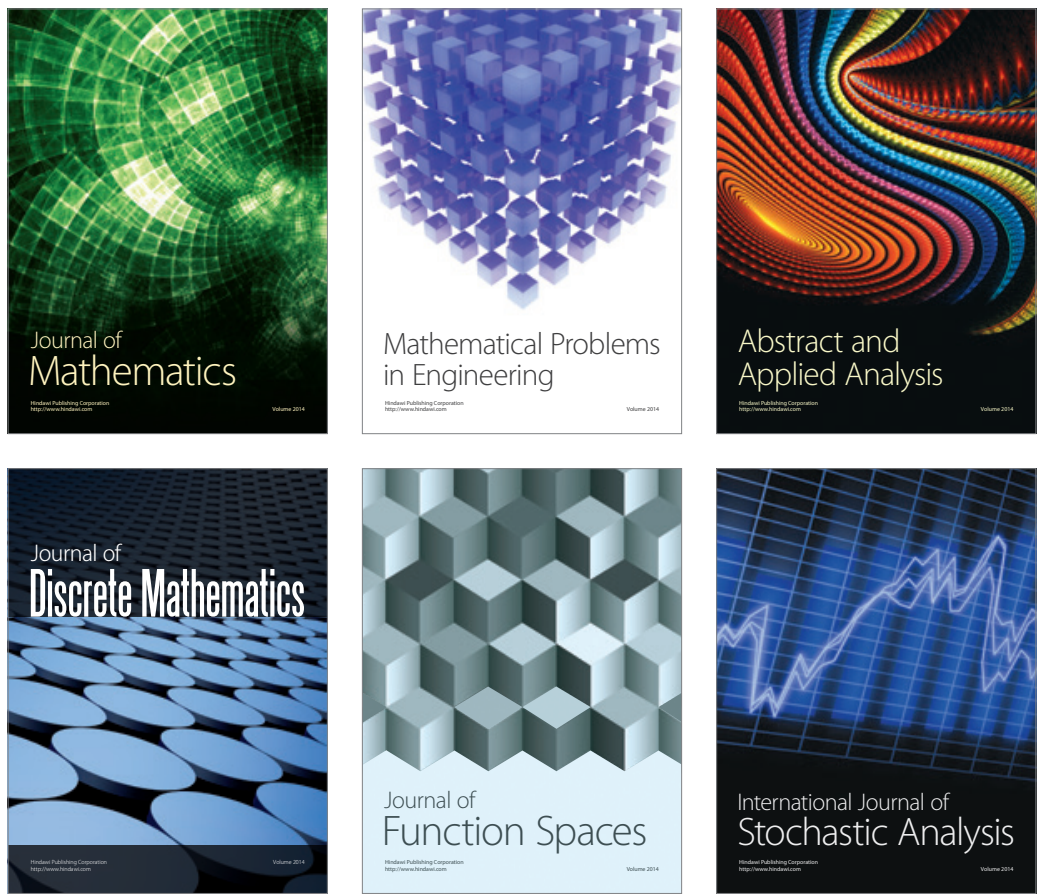

Journal of

Function Spaces

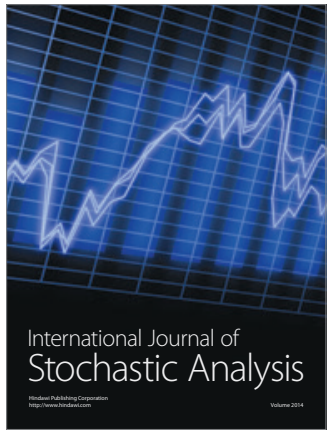

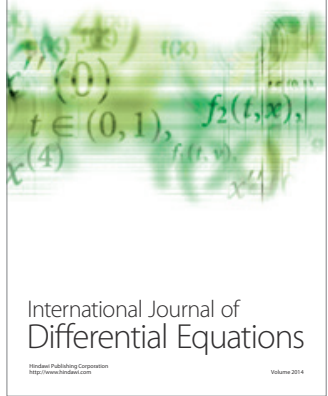
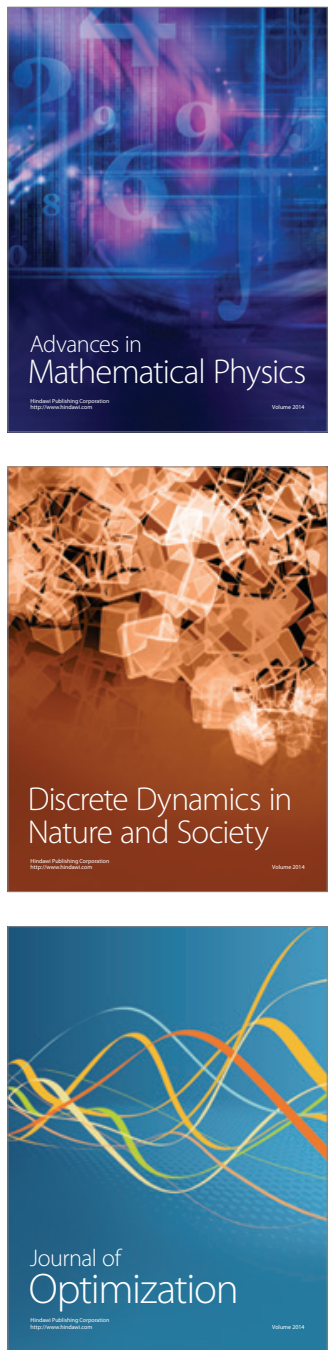